\title{
Counselling and adverse event management for patients with myelodysplastic syndromes undergoing azacitidine therapy: A practice standard for Canadian nurses
}

by Cindy Murray, Annie Wereley, Shannon Nixon, Carol Hua-Yung, Sarah von Riedemann and Sandra Kurtin, on behalf of the Canadian Nurses' Working Group on Azacitidine in MDS

\section{Abstract}

Azacitidine (5-azacytidine, VIDAZA ${ }^{*}$ ) is a disease-modifying agent that improves survival, reduces transfusion dependence, and reduces progression to acute myeloid leukemia in patients with higher risk myelodysplastic syndromes. Azacitidine injection is associated with characteristic adverse events (AEs) that must be managed in order for patients to stay on therapy and achieve optimal therapeutic outcomes. These AEs include injection-site reactions, cytopenias, and

\section{About the authors}

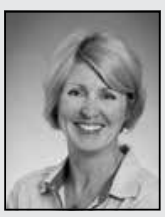

Cindy Murray, RN, NP-adult, Princess Margaret Hospital, University Health Network, Toronto, ON. Cindy.Murray@uhn.on.ca

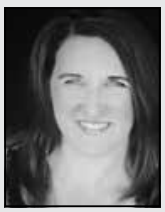

Annie Wereley, RN, The Ottawa Hospital, Ottawa, ON

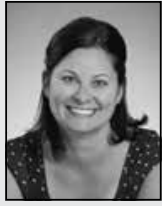

Shannon Nixon, RN, BSCN, Princess Margaret Hospital, University Health Network, Toronto, ON

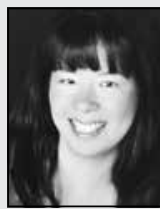

Carol Hua-Yung, RN, The Ottawa Hospital, Ottawa, ON

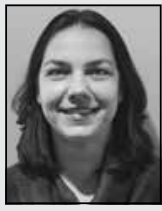

Sarah von Riedemann, MSc, SCRIPT, Toronto, ON

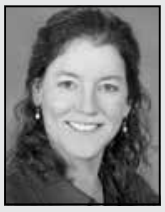

Sandra Kurtin, RN, MS, AOCN, ANP, The University of Arizona Cancer Center, Tucson, Arizona

On behalf of the Canadian Nurses' Working Group on Azacitidine in MDS

Mentor: Sandra Kurtin

Co-chairs: Carol Hua-Yung and Annie Wereley

Advisors: Nicole Crisp, Tammy DeGelder, Maggie Ford, Lucie Laporte, Cindy Murray, Shannon Nixon, Lollita Rahaman, Karen Roberts, Marg Mayer, and Jennifer Stephens gastrointestinal effects. Oncology nurses are uniquely positioned to provide patient support and counselling, thereby helping patients and their families set clear expectations for azacitidine therapy. This article presents a nursing standard designed to support Canadian oncology nurses in the key areas of counselling for patients initiating and continuing azacitidine, as well as nursing strategies for prevention and management of azacitidine-associated AEs. Many of the general principles discussed in this nursing standard can be applied broadly to many diseases and treatments.

\section{Background}

Myelodysplastic syndromes: definition and incidence

The myelodysplastic syndromes (MDS) are a group of disorders characterized by abnormal differentiation and maturation (dysplasia) of the hematopoietic stem cells, notably the myeloid cell lines. This leads to bone marrow failure, ineffective hematopoiesis, and peripheral cytopenias, including anemia, thrombocytopenia, and neutropenia. The early stages of MDS are frequently asymptomatic. As such, diagnosis of MDS is often incidental, during routine blood tests or physical examinations. However, over time MDS can cause significant morbidity (e.g., transfusion dependence, thrombocytopenia-related bleeding) and mortality (most commonly due to leukemic transformation or infectious disease complications) (Demakos \& Linebaugh, 2005).

MDS are primarily diseases of the elderly, with a median age at diagnosis of 76 years, and are more prevalent in men than in women (Buckstein et al., 2009). The exact incidence and prevalence of MDS are difficult to estimate due to under-diagnosis and under-recognition of the diseases. Buckstein et al. (2009) estimate the Canadian prevalence to be between 10,000 and 50,000 cases in people 65 years and older.

\section{Classification of MDS}

The myelodysplastic syndromes cover a wide clinical spectrum with many different subtypes. Traditionally, the subtypes were defined solely by the appearance and maturity of the blood and bone marrow cells. The first classification system used to define MDS was the French-American-British (FAB) system in 1976, but advances in cytogenetic and molecular knowledge have resulted in the refinement of $\mathrm{FAB}$ and the development of other classification systems (Haase et al., 2007). According to the World Health Organization (WHO) classification system, when blast counts in the marrow rise above $20 \%$, the disease is considered to have transformed to acute myeloid leukemia (AML) (Bennett, 2000). This occurs in approximately 40\% of MDS patients (Kurzrock, 2002).

Along with the WHO and FAB classifications, prognostic scoring systems such as the International Prognostic Scoring System (IPSS) are key to defining patient risk and setting management goals. IPSS scoring takes into account number of bone marrow blasts, karyotype, and presence of cytopenias to yield a numerical score from 0 to 3.5 that stratifies patients into four risk categories: Low (IPSS score 0 ), Intermediate-1 (0.5 to 1.0), Intermediate-2 (1.5 to 2.0), and High (2.5 and higher) (Greenberg et al., 1997). Higher IPSS scores are associated with increased risk of leukemic transformation (Greenberg et al., 1997), transfusion dependence (Balducci, 2006), and shorter median survival time (Greenberg et al., 1997). The IPSS was the first model predicting overall survival, but is currently being revised to incorporate age, performance status, serum ferritin levels, and bone marrow fibrosis (Greenberg et al., 2011). 


\section{Management of MDS}

The goals and choice of treatment in MDS are generally guided by the patient's IPSS score and other measures of disease risk, such as comorbidities, transfusion dependence, and levels of lactate dehydrogenase (LDH), a marker known to correlate with disease status (Kurtin \& Demakos, 2010). For patients with lower risk disease (Low and Intermediate-1 categories), therapy is usually aimed at enhancing hematopoiesis and alleviating symptoms through use of supportive measures such as blood transfusions, hematopoietic growth factors, and immunosuppressive agents. For patients with higher risk disease (Intermediate-2 and High categories), the additional goal, along with supportive care, is to prolong survival by modifying the course of the disease itself (Cheson et al., 2000). Until recently, the only disease-modifying option for MDS was hematopoietic stem cell transplantation from a human leukocyte antigen (HLA) matched donor (Gore, 2004). Success rates vary widely; depending on disease status and individual risk factors, anywhere from 30\% to $70 \%$ of patients undergoing transplantation can expect to experience a cure and survive long-term (Bartenstein \& Deeg, 2010). However, the advanced age of many MDS patients and a shortage of appropriate donors mean that transplants are not a viable option for most patients (Gore, 2004). Additionally, complications such as graft-versus-host disease are common, and treatment-related mortality can range from $34 \%$ (for non-myeloablative and reduced-intensity conditioning regimens) to $61 \%$ (for traditional myeloablative regimens) at five years post-transplant (Kindwall-Keller \& Isola, 2009).

Azacitidine. With the development of DNA hypomethylating agents such as azacitidine that have the capacity to alter the disease course, there has been a paradigm shift in the management of higher-risk MDS (List, Vardiman, Issa, \& DeWitte, 2004). Compared with conventional care regimens that consist of blood transfusion support with or without low-dose chemotherapy, azacitidine treatment in higher-risk MDS is associated with significantly improved survival, higher response rates, higher rates of transfusion independence, and delayed progression to AML (Fenaux et al., 2009).

Azacitidine is administered as a subcutaneous injection, and the most common adverse events (AEs) are myelosuppression and associated cytopenias, as well as gastrointestinal effects (nausea, vomiting) and injection-site reactions (VIDAZA Product Monograph, 2012). Cytopenias are most pronounced early in treatment, and the development of new cytopenias declines after the second azacitidine cycle (Santini et al., 2010).

In Canada, azacitidine is indicated for the treatment of adult patients who are ineligible for stem cell transplant and have higher risk MDS (Intermediate- 2 or High risk on the IPSS) or AML with $20 \%$ to $30 \%$ blasts and multi-lineage dysplasia according to the

\begin{tabular}{|l|l|}
\hline \multicolumn{2}{|l|}{ Table 1: Review of CANO/ACIO standards (CANO/ACIO, 2006) } \\
\hline $\begin{array}{l}\text { CANO/ACIO standards } \\
\text { of care }\end{array}$ & $\begin{array}{l}\text { Practice standards and competencies } \\
\text { for the specialized oncology nurse }\end{array}$ \\
\hline - Individualized and & - Comprehensive health assessment \\
holistic care & Supportive and therapeutic \\
- Family-centred care & relationships \\
- Self-determination and & - Management of cancer symptoms \\
decision-making & and treatment side effects \\
- Navigating the system & - Teaching and coaching \\
- Coordinated continuous & - Facilitating continuity of care/ \\
care & navigating the system \\
- Supportive therapeutic & - Decision-making and advocacy \\
relationship & Professional practice and leadership \\
- Evidence-based care & \\
- Professional care & \\
- Leadership & \\
\hline
\end{tabular}

WHO classification (Health Canada, 2010). The recent clinical practice guidelines of the Canadian Consortium on Evidence-Based Care in MDS support first-line use of azacitidine in this population (Buckstein, Yee, \& Wells, 2011). Additionally, azacitidine is being evaluated in clinical trials to determine whether it is useful in other indications and may be available on a compassionate-use basis in non-MDS conditions. In the U.S., the clinical experience with azacitidine has been longer (the FDA approved azacitidine in 2004, versus 2009 for Health Canada), and the indication in MDS differs. Specifically, in the U.S. there is no requirement for stem cell transplant ineligibility, and patient selection is based on FAB classifications rather than IPSS risk stratification (Health Canada, 2010; National Cancer Institute, 2011).

Mechanism of action of azacitidine. DNA hypermethylation is a common and constant process in pathogenesis of MDS and is associated with an increased risk of leukemic transformation (Kurtin \& Demakos, 2010). Effective suppression of DNA methylation requires continued DNA methyltransferase inhibition. Therefore, azacitidine should be given as repeated cycles for as long as the patient continues to benefit or until disease progression; a minimum of six cycles is recommended (Kurtin \& Demakos, 2010). The recommended dosing of azacitidine consists of seven consecutive days of azacitidine injections, followed by a 21-day rest period, for a total of 28 days in the cycle (VIDAZA Product Monograph, 2012). However, due to clinic schedules and other resource considerations, dosing on seven consecutive days is not possible in some centres. This has necessitated the development of non-standard dosing schedules such as 5-2-2: five days of injections, two days (usually the weekend) off, and then two more days of treatment. So far, there are no studies to provide evidence to show whether alternate dosing schedules affect the efficacy of azacitidine in the higher risk MDS population. In addition to its mechanism of action as a hypomethylating agent, azacitidine has inherent cytotoxic properties that necessitate special handling similar to procedures used for chemotherapeutic agents (e.g., preparation under hood, safe disposal as biohazard waste).

\section{Development of a nursing standard to support azacitidine use in MDS patients}

As administration of azacitidine is through daily subcutaneous injections and its AEs can be adequately managed, it is given in the ambulatory chemotherapy units of most cancer centres. However, there are some disadvantages to this delivery model. Nurses in the outpatient setting typically have a limited amount of time to spend with each patient and often see patients with a wide range of diagnoses. This model results in nurses being "less specialized," as they require a broader scope of knowledge and skills.

Additionally, because MDS is a rare disease and azacitidine a relatively new treatment option, nurses in smaller oncology clinics or community health care centres may have limited experience with azacitidine administration. Since the published nursing literature on patient counselling and AE management in azacitidine therapy is sparse, the nursing standard presented in this paper has been designed to address the gap by presenting consensus-based guidance that draws on the best practices of nursing experts.

\section{Process for standard development}

Working in collaboration with the pharmaceutical company that markets azacitidine in Canada, four leading oncology nurses from Canada (CH-Y, CM, SN, AW) and one from the United States (SK) met to develop and refine an outline of the key topics to be covered in the standard. These authors and a panel of expert oncology RNs from across Canada gathered at a consensus meeting in Edmonton, Alberta, in September 2010 to share best practices that could be incorporated into the standard. A consensus workshop method (brainstorming, clustering, naming) was used to generate a long list of possible recommendations and to group and refine the results. 
Following the meeting, draft versions of the standard were written and circulated to the authors and other panel members for feedback on content, wording and clinical applicability of the guidance. The standard was also circulated to nurses in the community who had not been part of the workshop panel for their input and questions. A literature search was conducted to identify any published evidence supporting or refuting the recommended best practices.

The guidance provided in this nursing standard was designed to align with two core standards of the Canadian Association of Nurses in Oncology/Association canadienne des infirmières en oncologie (CANO/ACIO): the Standards of Care and the Practice Standards and Competencies for the Specialized Oncology Nurse (Table 1). The final version of the standard was reviewed and accepted for endorsement by CANO.

\section{Content of standard}

This nursing standard focuses on two areas where nurses are uniquely positioned to provide effective support for azacitidine-treated patients: patient counselling and management of AEs. These two areas were chosen because they are central to the patient experience.

\section{Patient counselling}

In developing the patient-counselling standard (see the insert in this issue, part 1), five major themes emerged: general principles for counselling, informed consent, schedule and administration, support, and follow-up. The "Basic Principles" section of the standard was included as a reminder of best practices that apply more widely to the majority of patients that oncology nurses will encounter. These principles include elements related to obtaining a good patient history, providing appropriate levels of education, and setting reasonable expectations for disease management.

The other four themes are guidance categories that should be considered when patients are starting azacitidine therapy:

1) The "Informed Consent" section serves as a template for a discussion with patients about their understanding of their condition and its management. It is particularly important at this stage to explain the concept of continuous treatment and reinforce why it is important. Some patients will expect that once the treatment has "worked," they will be able to stop receiving it. Conversely, patients who fail to see a response within the first few cycles may think that treatment has failed and may not understand why they should continue receiving therapy. Patients also need to understand that azacitidine is not a cure for MDS. Because of the wide clinical spectrum of MDS and the differing management goals associated with different risk categories, it is important for patients to understand why azacitidine was chosen in their case, and why other patients they talk to could be receiving different treatment for their MDS.

2) The "Schedule and Administration" section of the standard is designed to help nurses give patients the tools they need to navigate their various appointments and tests. This is an area where printed materials such as calendars and patient diaries may be particularly useful. The section also includes guidance on how nurses can help patients understand what to expect at their visits, in terms of the process and the time required. Many patients are understandably apprehensive about receiving a new therapy, and unexpected occurrences (e.g., having to wait longer than anticipated, seeing nurses gowned up when not expecting it) can add to that anxiety. Clear communication about expectations and procedures can help alleviate some of the patient's worries.

3) The section on "Support" focuses on addressing patients' needs after they leave the injection clinic. These best practices are designed to help nurses assess patients' living circumstances and identify any educational or logistical assistance that could help them manage their condition and any AEs associated with their therapy.

4) Finally, the "Follow-up" section provides guidance on patient education about adverse events (AEs). Specifically, guidance on how to reduce the risk, what to watch for, and how to manage AEs if they do occur is included in this section.

\section{Adverse event (AE) management}

Because azacitidine is given in repeated cycles over a long period of time (usually at least six months, longer if the patient continues to benefit), it is of great importance to reduce the risk and impact of AEs so that the patient is willing and able to stay on therapy for as long as clinically appropriate (Santini et al., 2010). The intent with the AE management portion of the nursing standard (see the insert in this issue, part 2) was to focus on the AEs that are particular to azacitidine (e.g., injection-site reactions, cytopenias). Comprehensive guidance regarding nursing intervention for the more widely observed AEs is available elsewhere.

Injection-site reactions. Injection-site reactions (ISRs) and other skin events at the injection site are among the most common AEs with azacitidine use. They can take the form of erythema (about $40 \%$ of patients; Figure 1), red- or purplish-coloured, lumpy ISRs

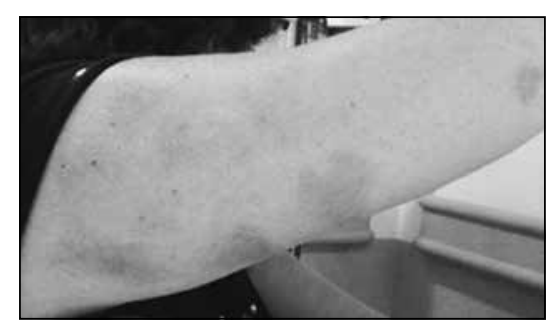

Figure 1: Typical injection-site reaction, 24 hours after azacitidine administration Photo courtesy of Cindy Murray, Princess Margaret Hospital, Toronto

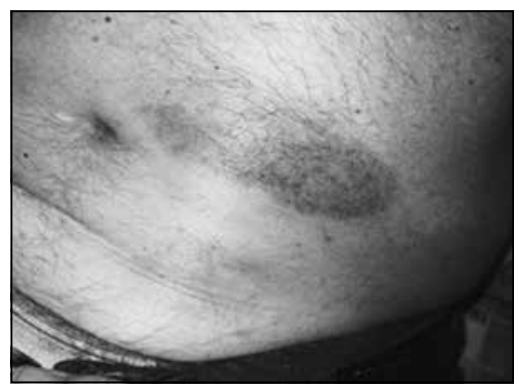

Figure 2: Hematoma after azacitidine injection in a patient with low platelet count Photo courtesy of Annie Wereley, The Ottawa Hospital
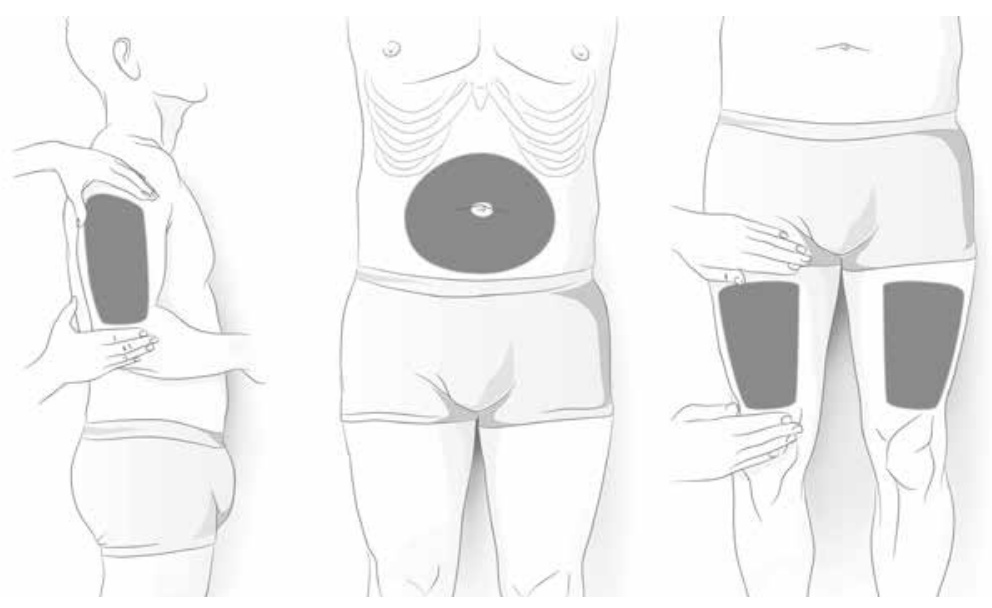

Figure 3: Appropriate injection sites Injection sites on the side of the arm, the abdomen, and the thighs 
( 30\%), pain at the injection site ( 20\%), or hematomas or less severe bruising ( 10\%; Figure 2) (VIDAZA Product Monograph, 2012). In rare cases, ISRs can lead to pigmentation changes at and near the injection site. Although these reactions are rarely severe in clinical terms (the majority are Grade 1 or 2 ), they can be very uncomfortable and distressing for patients.

The guidance in the nursing standard has been designed to give nurses practical tips for establishing appropriate patient expectations about ISRs, reducing the severity of any reactions that occur, and providing relief and support after the injection. One of the key methods for reducing the risk and severity of ISRs is proper injection technique, appropriate selection and rotation of injection sites (Figure 3), small injection volumes (Demakos \& Linebaugh, 2005), and an "air sandwich" (Figure 4) in the syringe to ensure full administration of the desired volume (Kurtin \& Demakos, 2010). The limits for total volume that can be given in a single injection will vary from institution to institution but, generally, range up to $4 \mathrm{~mL}$. Please consult your own institution's policy for specific guidance.

Techniques for reducing ISR symptoms are drawn mainly from anecdotal evidence and clinical experience (Demakos \& Linebaugh, 2005), although a small case series has shown the efficacy of evening primrose oil (Platzbecker, Aul, Ehninger, \& Giagounidis, 2010) in reducing the itch and discomfort associated with azacitidine-related ISRs. Although a recent study in multiple sclerosis patients using a subcutaneously injected therapy failed to find any impact of oral antihistamines on the frequency of ISRs (Pardo, Boutwell, Conner, Denney, \& Oleen-Burkey, 2010), many azacitidine-treated patients report feeling some symptom relief with oral, over-thecounter antihistamines taken either before or after the injection.

Cytopenias. Because many MDS patients will have one or multiple cytopenias even before initiation of azacitidine treatment (Santini et al., 2010), the further drops in blood counts that often occur with therapy must be carefully monitored and managed. In key clinical trials of azacitidine, $70 \%$ of patients experienced thrombocytopenia, while $66 \%$ had some degree of neutropenia and 51\% experienced anemia (VIDAZA Product Monograph, 2012). In clinical practice, cytopenias are generally defined as blood counts that fall below the normal ranges noted in Table 2. Although these thresholds can be clinically useful for identifying cytopenias, single measurements of blood counts are not the only factor that should be considered. Trends in blood counts over time should also be tracked in order to identify patients at risk of becoming cytopenic.

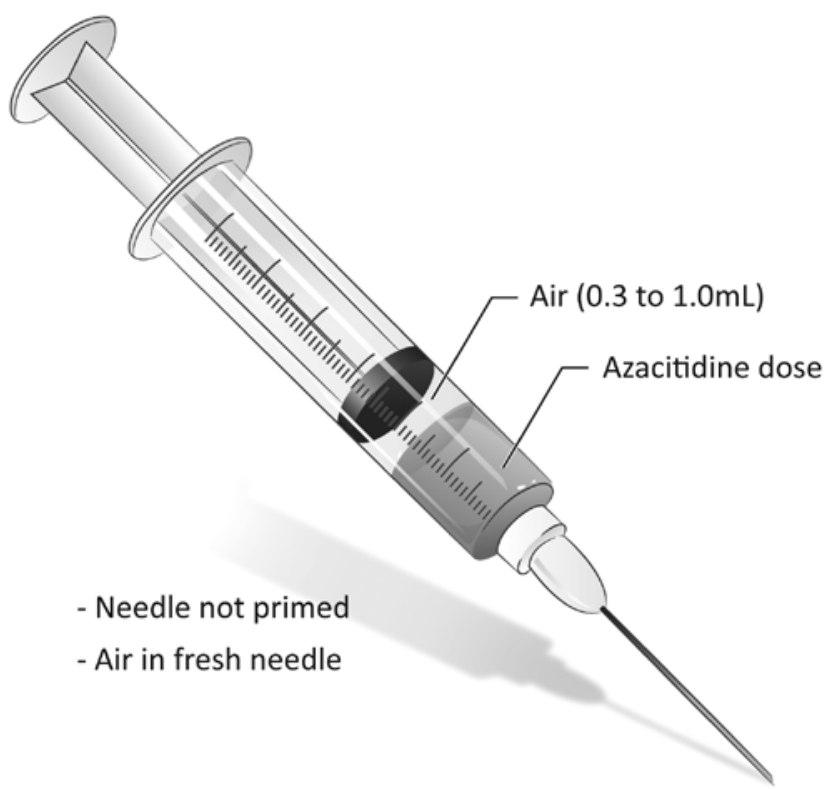

Figure 4: “Air sandwich" in syringe

\begin{tabular}{|l|l|}
\hline \multicolumn{2}{|l|}{ Table 2: Clinical definitions of normal blood parameters } \\
\hline Test & $\begin{array}{l}\text { Range (normal low } \\
\text { to normal high) }\end{array}$ \\
\hline Hemoglobin & $\begin{array}{l}\text { Men: } 130-180 \mathrm{~g} / \mathrm{L} ; \\
\text { women: } 120-160 \mathrm{~g} / \mathrm{L}\end{array}$ \\
\hline White blood cells (WBCs)/leukocytes & $3.5-10.5 \times 10^{9} / \mathrm{L}$ \\
\hline Absolute neutrophil count (ANC) & $1.5-8.0 \times 10^{9} / \mathrm{L}$ \\
\hline Platelets & $140-440 \times 10^{9} / \mathrm{L}$ \\
\hline
\end{tabular}

Note: Normal ranges vary slightly around the diagnostic test; these figures are given solely as a rough guide.

It is important for nurses and patients to appreciate that cytopenias are an expected consequence of azacitidine therapy. Patients should be reassured that although counts may drop at first with treatment, the numbers should return with time if the patient responds to the drug (Santini et al., 2010). Patients should be counselled on when to report their cytopenic symptoms and how to reduce the risk of complications (e.g., infections for neutropenic patients, bruising/bleeding for thrombocytopenic patients). It is also important to ensure regular blood work follow-up and timely use of hematopoiesis-stimulating agents (e.g., erythropoietin, granulocyte-stimulating factor) if clinically indicated (Kurtin \& Demakos, 2010).

Gastrointestinal events. Gastrointestinal AEs are common with azacitidine therapy; almost half of patients will experience nausea, and one-quarter will experience vomiting (VIDAZA Product Monograph, 2012). This places azacitidine in the "moderately emetogenic" category of anti-cancer agents. As such, pre-medication with anti-emetics is recommended before each administration (Demakos \& Linebaugh, 2005; National Comprehensive Cancer Network, 2011). Constipation is also common, occurring in about half of azacitidine-treated patients. Routine use of anti-emetic medication may contribute to this incidence (Demakos \& Linebaugh, 2005). Because the gastrointestinal events commonly observed with azacitidine therapy do not differ significantly in quality or management from those seen with other anti-cancer agents, nurses are encouraged to refer to more general guidelines such as those of Cancer Care Ontario, the Oncology Nursing Society, or the National Comprehensive Cancer Network for further guidance on gastrointestinal event management that is applicable to patients receiving azacitidine.

\section{Conclusions}

This nursing standard was designed to provide clinically useful guidance on patient counselling and $\mathrm{AE}$ management to nurses across Canada who assist with azacitidine treatment for patients with MDS, whether in oncology clinics, community medical centres, or other practice locations. The standard should be updated or revised to reflect the ongoing refinement of dosing schedules, our advancing knowledge of patient counselling techniques, and any new options for AE management. Once the standard has been in clinical use for some time, it could be valuable to study its impact on nursing practice and patient experience. It is hoped that with this standard, care of the azacitidine-treated patient can be enhanced, leading to better quality of life and improved treatment outcomes for patients.

\section{Acknowledgement}

This project was supported by an unrestricted educational grant from Celgene Inc. All authors were fully responsible for the writing, content, and final approval of the manuscript. 


\section{Application of the nursing standard: Case study}

The following case study is based on a real-life patient (some details have been modified) and provides an example of how the nursing standard can be applied in practice.

\section{Clinical narrative \\ Ms. KR was a 68-year-old woman diagnosed with MDS in early 2010. At the time of diagnosis, her hemoglobin was 67 $\mathrm{g} / \mathrm{L}$, white blood cell count was $5.3 \times 10^{9} / \mathrm{L}$, and platelet count was $8 \times 10^{9} / \mathrm{L}$. Her bone marrow aspirate showed multiple high-risk cytogenetic abnormalities and $10 \%$ blasts. According to IPSS (Greenberg et al., 1997), these disease characteristics placed her in an Intermediate-2 risk category. Because of her relatively young age, high-risk cytogenetics and the absence of significant comorbidities, her hematologist recommended an allogeneic stem cell transplant (ASCT). After consultation with the team, she decided against ASCT because she was concerned about the side effects of transplantation and their impact on her ability to travel and other aspects of her lifestyle.}

Ms. KR started treatment with azacitidine in April 2010. At this point she was receiving platelet transfusions twice weekly and packed cell transfusions once weekly. She experienced large hematomas at the azacitidine injection sites. One injection site became infected and she presented with a moderately severe cellulitis.

\section{Nursing interventions and application of the standard}

It is important for the nurse to build on the background information provided for Ms. KR, starting with the basic principles provided in the standard. A thorough history revealed that Ms. KR had not experienced any major illnesses in her life. On reflection, she could recite a basic understanding of MDS from her previous appointments with her hematologist. She had never been hospitalized and had limited experience with friends and family being ill or hospitalized. It was, therefore, difficult for her to describe her coping strategies related to her health. However, it was clear that she would require lots of time for education around her disease and its treatment, as she preferred to learn by asking questions. English was her first language and did not provide a barrier to her learning. Her main support system was her mother, who lived with her and accompanied her to clinic visits, so it was important to include her mother in any teaching with Ms. KR.

It is important for the nurse to be familiar with azacitidine-related injection site reactions (as described in the "reducing AEs" section of the standard) and be able to distinguish between an infected site and an expected reaction in order for an infection to be treated with appropriate antibiotics. Nurses should also introduce and then reinforce tips for minimizing the risk of ISRs, such as avoiding lotion or tight clothing on the injection site.

As described in the counselling section of the standard, it is very important for nurses to emphasize the predictability of cytopenias associated with the treatment during the initial few cycles. It was important for the nurses in this case to reassure Ms. KR that the drop in her blood counts was most likely transient and could be managed. They also encouraged her to focus on remaining optimistic and hopeful about her treatment.

After two cycles of azacitidine treatment, Ms. KR became transfusion independent and began to travel again. She continued monthly cycles of azacitidine and experienced a couple of infectious complications, including herpes zoster and pneumonia.

Her physician continued to recommend ASCT, but Ms. KR declined.

By November 2010 Ms. KR's hemoglobin and platelet counts had begun to drop and she required transfusions again.

In January 2011, a bone marrow aspirate revealed her MDS had transformed to acute leukemia with 50\% blast cells. She was admitted to hospital with pneumonia in early 2011 and received two induction attempts with chemotherapy. The leukemia did not respond to treatment, and she passed away in the summer of 2011.
As discussed in the AE section of the standard, nurses should educate patients about reportable signs of infection so they can be treated early to minimize the associated morbidity. Ms. KR alerted her health care team and was started on treatment early; she, therefore, did not develop painful post-herpetic neuralgia or serious complications of pneumonia.

As per the "informed consent" theme in the counselling standard, nurses administering the injections to Ms. KR reinforced the fact that azacitidine can modify the course of MDS, but does not offer a cure, and that transplantation remains the only treatment option that could potentially lead to a cure. Because of the high-risk nature of her disease, the chance of her MDS transforming to acute leukemia was quite high. Nurses should be aware that it can be challenging to present this information to patients without sounding overly insistent. The goal should be to appreciate the patient's concerns and rationale, and to support their choices with the best information and care possible.

Ms. KR and her mother were understandably very distraught and clearly understood her disease was progressing. They both required emotional support from the nurses, in particular Ms. KR's mother.

Summary

Key clinical and lifestyle outcomes with azacitidine treatment: Key nursing interventions:

- Reduced transfusion requirements

- Ability to travel and enjoy improved quality of life
- Thorough patient history

- Evaluation of appropriate teaching level and methods

- Consideration of family and lifestyle priorities

- Identification and management of AEs

- Ongoing counselling about treatment options 
Balducci, L. (2006). Transfusion independence in patients with myelodysplastic syndromes: Impact on outcomes and quality of life. Cancer, 106(10), 2087-2094.

Bartenstein, M. \& Deeg, H.J. (2010). Hematopoietic stem cell transplantation for MDS. Hematology/Oncology Clinics of North America, 24(2), 407-422.

Bennett, J. M. (2000). World Health Organization classification of the acute leukemias and myelodysplastic syndrome. International Journal of Hematology, 72(2), 131-133.

Buckstein, R., Jang, K., Friedlich, J., Zhang, L., Reis, M., Chesney, A., \& Wells, R. A. (2009). Estimating the prevalence of myelodysplastic syndromes in patients with unexplained cytopenias: A retrospective study of 322 bone marrows. Leukemia Research, 33(10), 1313-1318.

Buckstein, R., Yee, K., \& Wells, R.A.; Canadian Consortium on Evidence-based Care in MDS. (2011). 5-Azacytidine in myelodysplastic syndromes: A clinical practice guideline. Cancer Treatment Reviews, 37(2), 160-167. Epub 2010 Jun 29.

Canadian Association of Nurses in Oncology/Association canadienne des infirmières en oncologie. (2006). Practice Standards and Competencies for the Specialized Oncology Nurse.

Cheson, B.D., Bennett, J.M., Kantarjian, H., Pinto, A., Schiffer, C.A., Nimer, S.D., ... Greenberg, P.L. (2000). Report of an international working group to standardize response criteria for myelodysplastic syndromes. Blood, 96(12), 3671-3674.

Demakos, E.P. \& Linebaugh, J.A. (2005). Advances in myelodysplastic syndrome: Nursing implications of azacitidine. Clinical Journal of Oncology Nursing, 9(4), 417-423.

Fenaux, P., Mufti, G.J., Hellström-Lindbert, E., Santini, V., Finelli, C., Giagounidis, A., ... Silverman, L.R., for the International Vidaza High-Risk MDS Survival Study Group. (2009). Efficacy of azacitidine compared with that of conventional care regimens in the treatment of higher-risk myelodysplastic syndromes: A randomised, open-label, phase III study. The Lancet Oncology, 10(3), 223-232.

Gore, S. (2004). Classification, treatment goals, and management principles for myelodysplastic syndromes. Cancer Control, 11(6 suppl), 3-6.

Greenberg, P., Cox, C., LeBeau, M. M., Fenaux, P., Morel, P., Sanz, G., ... Bennett, J. (1997). International scoring system for evaluating prognosis in myelodysplastic syndromes. Blood, 89(6), 2079-2088.

Greenberg, P., Tuechler, H., Schanz, J., Sole, F., Bennett, J.M., GarciaManero, G., \& Haase, D. (2011). Revised international prognostic scoring system (IPSS-R), developed by the international prognostic working group for prognosis in MDS (IWG-PM). Leukemia Research, 35(s6), Abstract 14.

Haase, D., Germing, U., Schanz, J., Pfellstoker, M., Hildebrandt, B., Kundgen, A., ... Steidl, C. (2007). New insights into the prognostic impact of the karyotype in MDS and correlation with subtypes: Evidence from a core dataset of 2,124 patients. Blood, 110(13), 4385-4395.

Health Canada. Summary basis of decision for azacitidine (PrVIDAZA ${ }^{\varpi}$. (2010). Retrieved from http://www.hc-sc.gc.ca/ dhp-mps/prodpharma/sbd-smd/drug-med/sbd_smd_2010_ vidaza_127108-eng.php

Kindwall-Keller, T., \& Isola, L.M. (2009). The evolution of hematopoietic SCT in myelodysplastic syndrome. Bone Marrow Transplantation, 43(8), 597-609. Epub 2009 Mar 2.

Kurtin, S., \& Demakos, E.P. (2010). An update on the treatment of myelodysplastic syndromes. Clinical Journal of Oncology Nursing, 14(3), E29-E44.

Kurzrock, R. (2002). Myelodysplastic syndrome overview. Seminars in Hematology, 39(3, Suppl. 2), 18-25.

List, A.F., Vardiman, J., Issa, J-P. J., \& DeWitte, T.M. (2004). Myelodysplastic syndromes. Hematology: American Society of Hematology Education Program, 297-317.

National Cancer Institute. (2011). FDA Approval for Azacitidine. Retrieved from www.cancer.gov/cancertopics/druginfo/ fda-azacitidine

National Comprehensive Cancer Network (NCCN). (2011). NCCN Clinical Practice Guidelines in Oncology: Myelodysplastic Syndromes. V.2. Retrieved from http://www.nccn.org/ professionals/physician_gls/pdf/mds.pdf

Pardo, G., Boutwell, C., Conner, J., Denney, D., \& Oleen-Burkey, M. (2010). Effect of oral antihistamine on local injection site reactions with self-administered glatiramer acetate. Journal of Neuroscience Nursing, 42(1), 40-46.

Platzbecker, U., Aul, C., Ehninger, G., \& Giagounidis, A. (2010). Reduction of 5-azacitidine induced skin reactions in MDS patients with evening primrose oil. Annals of Hematology, 89(4), 427-428. Epub 2009 Aug 28.

Santini, V., Fenaux, P., Mufti, J., Ellström-Lindbert, E., Silverman, L.R., List, A., ... Beach, C.L. (2010). Management and supportive care measures for adverse events in patients with myelodysplastic syndromes treated with azacitidine. European Journal of Haematology, 85(2), 130-138.

VIDAZA $^{\star}$ (azacitidine for injection) Product Monograph. Celgene Inc., Mississauga, Ontario, Canada; May 17, 2012. 